\section{Double Lumen Endotracheal Tube for Percutaneous Tracheostomy?}

\section{To the Editor:}

We read with great interest the article by Vargas et $\mathrm{al}^{1}$ on their in vitro study of the flow resistance of a double lumen endotracheal tube (DLET) suggested as an alternative to a standard orotracheal tube for ventilation during percutaneous tracheostomy. However, we feel some aspects deserve further comments. They made a comparison of flow resistance among the different standard-size endotracheal tubes and the DLET during fiberoptic bronchoscope insertion, remarking upon the presence of a well known increased flow resistance when the fiberoptic bronchoscope is inserted in standard devices. Although the authors carried out extensive work on the physical properties of the DLET in vitro, as we can clearly read in the abstract and text, the title refers to an in vivo clinical procedure. Moreover, even if they show relevant data, they extend their conclusions to clinical practice. The final judgment on the use of the device in vivo, based only on the in vitro experimental study, does not reflect the study's data.

Other comments based on our clinical experience are then necessary. The first, which concerns in vivo experimentation, is about tube positioning. We agree that the tube can be safely positioned with a tube exchanger (airway exchange catheter [AEC]), but a small one (outer diameter) is needed due to the distal DLET's elliptic shape (Fig. 1). For the same reason, DLET slippage on the AEC can result in further difficulties: when the DLET is inserted with the AEC, the elliptic shape defines 2 semilunar lateral areas exceeding the AEC profile (Fig. 1, blue areas). These areas could impact anatomical structures with glottic damage and bleeding if the DLET is positioned with only the AEC.

In our clinical practice, we use a small diameter tube (5-mm inner diameter) that can be safely positioned with the AEC without laryngoscopy, as we have described previously. ${ }^{2,3}$ We know that the resistances in the smaller diameter tube are increased, but they affect only the mechanical ventilator in the inspiratory phase. In the expiratory phase, the flow follows the physiological path because the tube is uncuffed. This system offers the greatest vision of the operatory field without any interference with

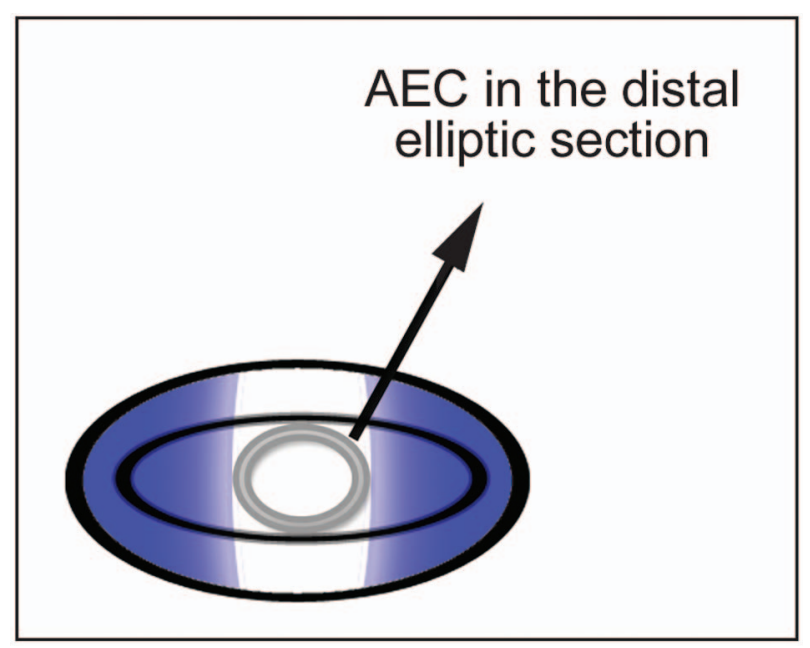

Fig. 1. Airway exchange catheter (AEC) in the distal elliptic section of a double lumen endotracheal tube.

ventilation or surgery during the entire procedure.

\section{Fausto Ferraro MD \\ Pierluigi Fusco MD \\ Lucia Marullo MD \\ Annarita Torino MD Naples, Italy}

Department of Anesthesiological, Surgical and Emergency Sciences Second University of Naples

The authors have disclosed no conflicts of interest.

DOI: $10.4187 /$ respcare.03911

\section{REFERENCES}

1. Vargas M, Servillo G, Tessitore G, Aloj F, Brunetti I, Arditi E, et al. Double lumen endotracheal tube for percutaneous tracheostomy. Respir Care 2014;59(11):1652 1659.

2. Ferraro F, Capasso A, Troise E, Lanza S, Azan G, Rispoli F, Anello CB. Assessment of ventilation during the performance of elective endoscopic-guided percutaneous tracheostomy: clinical evaluation of a new method. Chest 2004;126(1):159-164.

3. Ferraro F, Marfella R, Petruzzi J, Torino A, d'Elia A, Lettieri B. Translaryngeal open ventilation for percutaneous endoscopic tracheostomy. Br J Anaesth 2014;113(1) 189-190.

\section{Double Lumen Endotracheal Tube for Percutaneous Tracheostomy- Reply}

\section{In Reply:}

We read with interest the comments of Ferraro and colleagues regarding our paper evaluating a double lumen endotracheal tube (DLET) for percutaneous tracheostomy. ${ }^{1}$ The title of our paper introduces for the first time the DLET and its potential use during percutaneous tracheostomy. We specified in the abstract and text that this article was an in vitro evaluation of the DLET. The title of a scientific paper should describe the subject matter of an article, including appropriate key words for indexing, to attract potential readers' attention, especially in electronic databases. Ferraro and colleagues question the fact that our conclusion did not reflect the study's data. In our article, we concluded that use of the DLET during percutaneous tracheostomy did not impose an excessive increase in airway resistance, which is consistent with the data obtained during evaluation with continuous flow and mechanical ventilation. Furthermore, in the last sentence of our conclusion, we used the verb "may" to suggest the possibility of additional safety when using the DLET during percutaneous tracheostomy.

Ferraro and colleagues claimed that our paper over-reported in vitro data implying effects on clinical practice, but they considered it appropriate to comment on possible in vivo problems during DLET positioning. They raised concerns regarding potential dif- 
ficulties passing even a small tube exchanger through the DLET. In our article, we hypothesized that, in clinical practice, the positioning of the DLET may be safely performed with an appropriate tube exchanger. ${ }^{1}$ However, we clearly reported that the crosssectional diameter and area of the DLET were similar to those of a conventional endotracheal tube (ETT) even if the lower lumen had an elliptical shape. Furthermore, in our recent in vivo paper ${ }^{2}$ on the feasibility of DLET during percutaneous tracheostomy, we used a common tube exchanger to place the DLET without any difficulties or anatomical damage. Additionally, patients can be intubated with the DLET in a conventional manner or using a fiberoptic bronchoscope.

Ferraro and colleagues suggest an alternative clinical procedure for ventilation during percutaneous tracheostomy, already tested in vivo. ${ }^{3}$ They reported on the use of a small (5-mm internal diameter) uncuffed tube, claiming that it offered a better bronchoscopic vision without any interference with ventilation and percutaneous tracheostomy procedures. ${ }^{3}$ This method seems to guarantee oxygenation rather than ventilation during percutaneous tracheostomy because it resulted in an increased $\mathrm{P}_{\mathrm{aCO}}$. The improvement in ventilation using the DLET during percutaneous tracheostomy has now been reported in patients. ${ }^{2}$ Although the method proposed by Ferraro et $\mathrm{al}^{3}$ is feasible, it increases the $\mathrm{P}_{\mathrm{aCO}}$ and reduces the arterial $\mathrm{pH}$ similar to other conventional ETTs. In our recent in vivo study, ${ }^{2}$ using the DLET during percutaneous tracheostomy was not associated with any variation in gas exchange, acid/base balance, or airway pressure. Furthermore, the DLET may introduce additional advantages over conventional ETTs during percutaneous tracheostomy: it may reduce/eliminate the risk of accidental extubation and aspiration with its distal cuff, preserve the fiberoptic bronchoscope, and protect the posterior tracheal wall.

Maria Vargas MD

Giuseppe Servillo MD

Gaetano Tessitore MD

Department of Neuroscience and

Reproductive and

Odontostomatological Sciences

University of Naples "Federico II"

Naples, Italy

Fulvio Aloj MD

Anaesthesia and Intensive Care Unit Istituto di Ricovero e Cura a Carattere

Scientifico Neuromed Pozzilli, Italy

Iole Brunetti MD

Enrico Arditi MD

Dorino Salami MD

Department of Anesthesia and Intensive Care

Istituto di Ricovero e Cura a

Carattere Scientifico

Azienda Ospedaliera Universitaria

San Martino IST

University of Genoa

Genoa, Italy

Robert M Kacmarek PhD RRT FAARC Department of Anesthesiology and Critical Care Department of Respiratory Care
Massachusetts General Hospital Boston, Massachusetts

Paolo Pelosi MD

Department of Surgical Sciences and Integrated Diagnostics

Department of Anesthesia and Intensive Care

Istituto di Ricovero e Cura a Carattere Scientifico

Azienda Ospedaliera Universitaria San Martino IST University of Genoa Genoa, Italy

Support was provided solely by institutional and/or departmental sources. Dr Kacmarek discloses relationships with Covidien, Venner Medical, and Maquet. The other authors have disclosed no conflicts of interest.

DOI: $10.4187 /$ respcare.04009

\section{REFERENCES}

1. Vargas M, Servillo G, Tessitore G, Aloj F, Brunetti I, Arditi E, et al. Double lumen endotracheal tube for percutaneous tracheostomy. Respir Care 2014;59(11):16521659.

2. Vargas M, Pelosi P, Tessitore G, Aloj F, Brunetti I, Arditi E, et al. Percutaneous dilatational tracheostomy with a double lumen endotracheal tube: a comparison of feasibility, gas-exchange and airway pressures. Chest 2014 [Epub ahead of print] doi: 10.1378/chest.14-1465.

3. Ferraro F, Marfella R, Petruzzi J, Torino A, d'Elia A, Lettieri B. Translaryngeal open ventilation for percutaneous endoscopic tracheostomy. Br J Anaesth 2014;113(1):189190. 